\title{
Volumetric bone changes around dental implants the use of 3D image superimposition
}

IMPLANT THERAPY OUTCOMES, PERI-IMPLANT BIOLOGY ASPECTS

\section{E. Vilarinho ${ }^{1}$, A. Correia ${ }^{2,5 *}$, A. Vigo ${ }^{3}$, N. Viriato Ramos ${ }^{5}$, M. Vaz $^{4,5}$, R. Shinkai ${ }^{1}$}

${ }^{1}$ Faculty of Dental Medicine, Pontificial Catholic University of Rio Grande do Sul (PUCRS) - Brazil

Institute of Health Sciences (Viseu); Centre for Interdisciplinary Research in Health ; Universidade Católica Portuguesa - Portugal 3nstitute of Mathematics and Statistics, Dept Statistics, Federal University of Rio Grande do Sul - Brazil

Institute of Science and Innovation in Mechanical and Industrial Engineering (INEGI), University of Porto - Portugal * Presenting auther

\section{Abstract}

This research focus on a dental informatics protocol to measure volumetric changes in bone level around dental implants. CBCT \& Digital Periapical Radiography were used after implantation, 12 and 24 months of functional loading and $3 D$ reconstructions used to do a volumetric analysis $.2 D / 3 D$ analysis estimated that an increase of $1 \mathrm{~mm}$ of linear $B L$ was associated with a mean volumetric $B L$ of $\approx 14 \mathrm{~mm}^{3}(p<0.001)$.

\section{Background and Aim}

- Long-term success of implant treatment depends on the integrity of bone-implant interface. Besides clinical features, imaging exams are used to monitor changes in this area.

- 2D images are commonly used but they have distortions and generate image superimposition between buccal and lingual bone.

- Development of CBCT in Dentistry provide a detailed volumetric reconstruction (3D), of a region of interest. Afterwards,

superimposition techniques may be used to detect volume changes.

AIM: To present a dental informatics methodology to measure volumetric changes in the bone level around dental implants.

\section{Methods and Materials}

- Sample: 46 dental implants ( $6 \mathrm{~mm}$ length / $4.1 \mathrm{~mm}$ diameter) placed in posterior maxilla and mandible in 20 patients. All implants received screw-retained single crowns.

- CBCT \& Digital Periapical Radiography: after implantation (T0), 12 and 24 months of functional loading (T1 \& T2).

- 3D reconstructions of bone perimeter closest to the implant were developed, superimposed and volumetrically measured.

- Linear measures of bone levels were made in periapical images.

- Multilevel regression model tested volumetric and linear bone loss (BL).

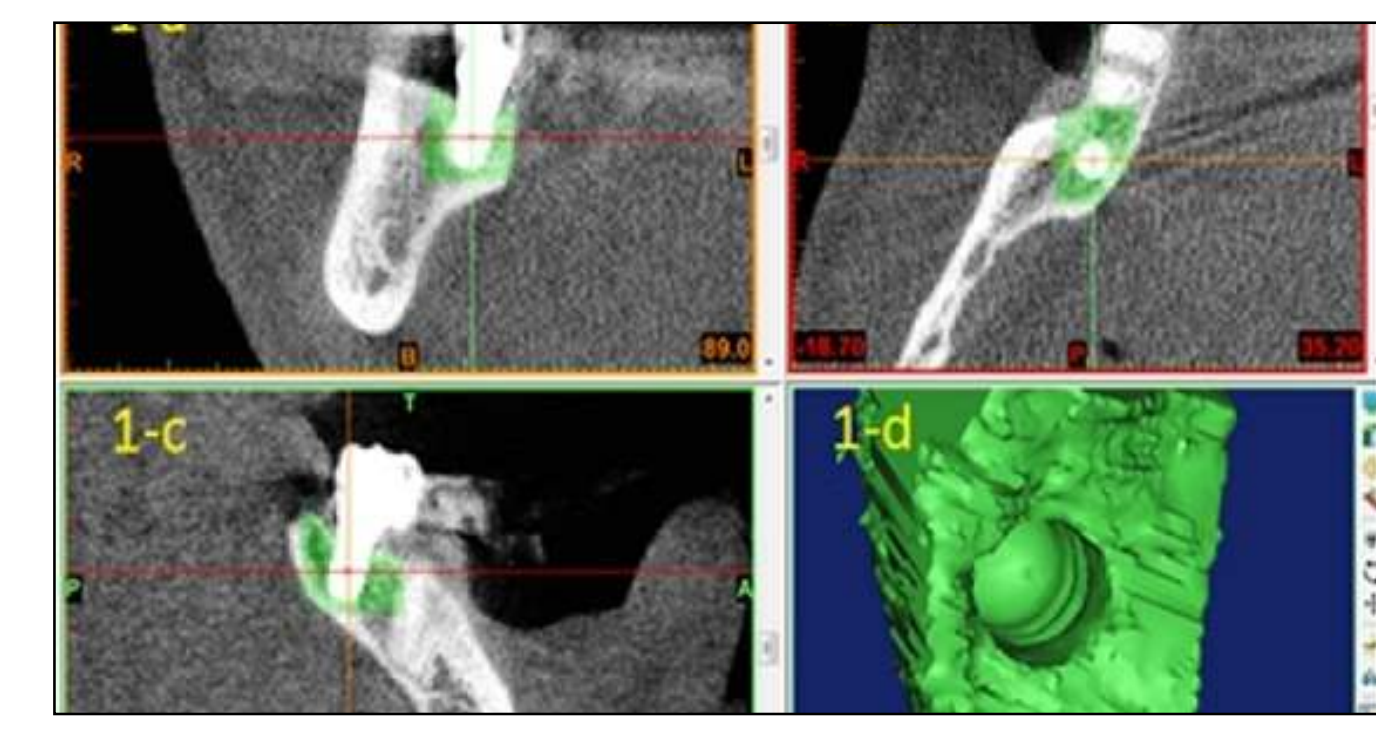

Fig. 1: Mimics $₫$ image, with bone region selected around the implant, checking that only the bone tissue is selected in 3D reconstruction.

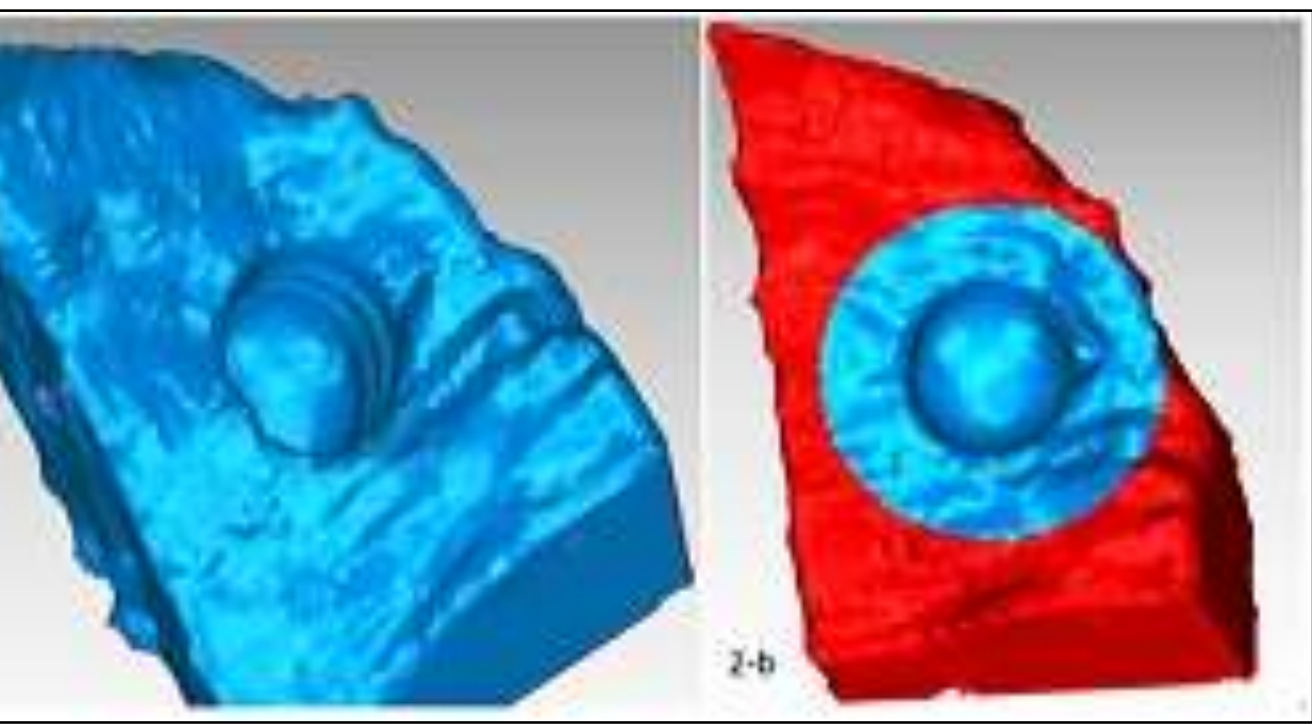

Fig. 2: STL image of bone tissue imported into Geomagic with selected image with clo
implant (right)

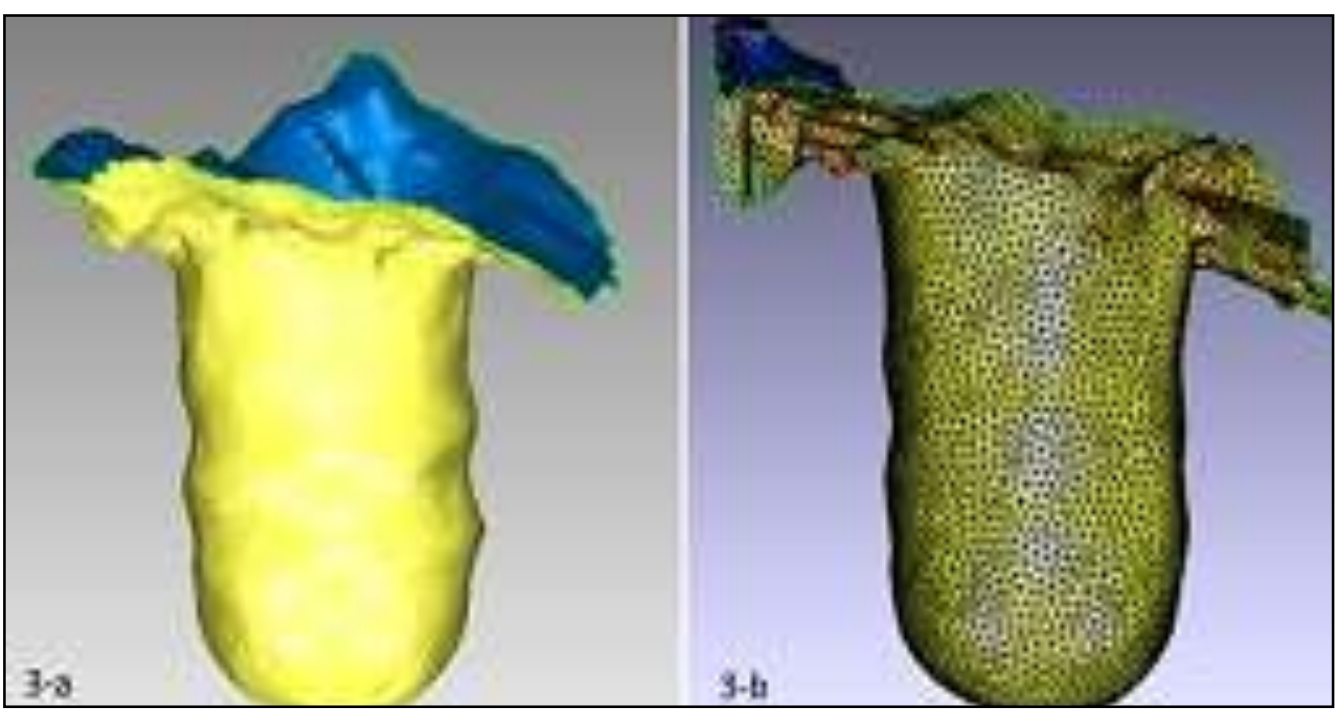

Fig. 3: a) Bone perimeter around selected implant, after removal of area of no interest for measurement. b) mesh refinement, for better image superimposition.
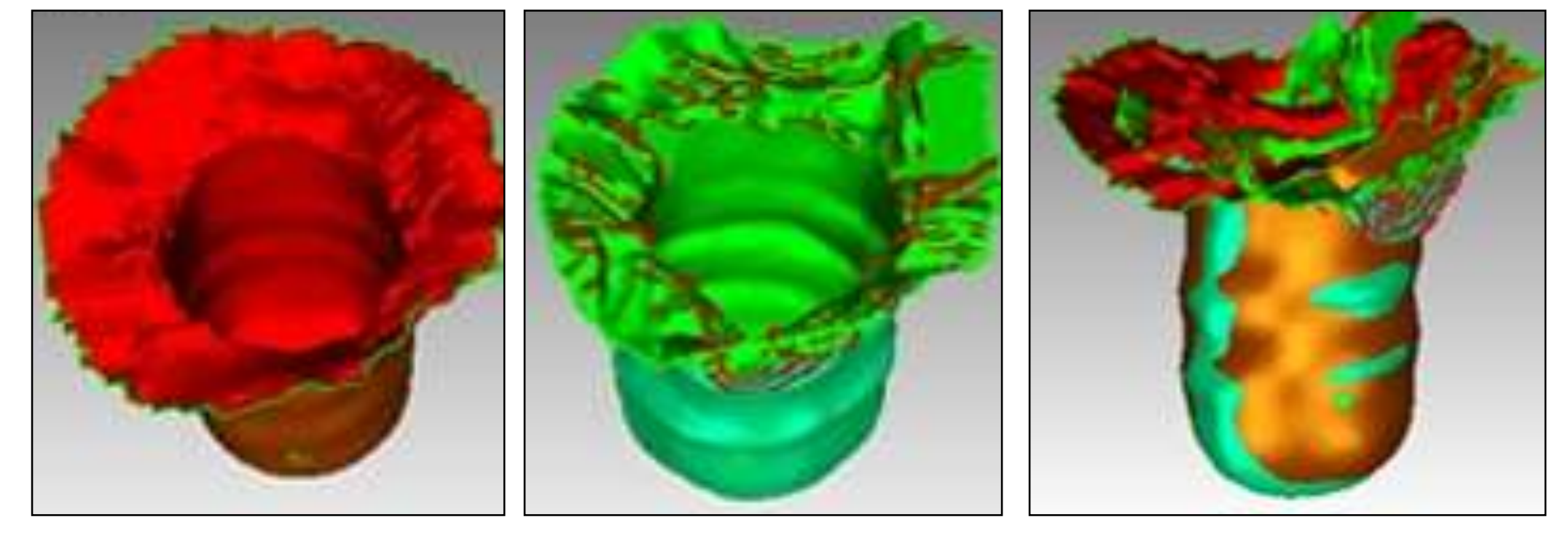

Fig. 4: Images at different times superimposed by alignmen

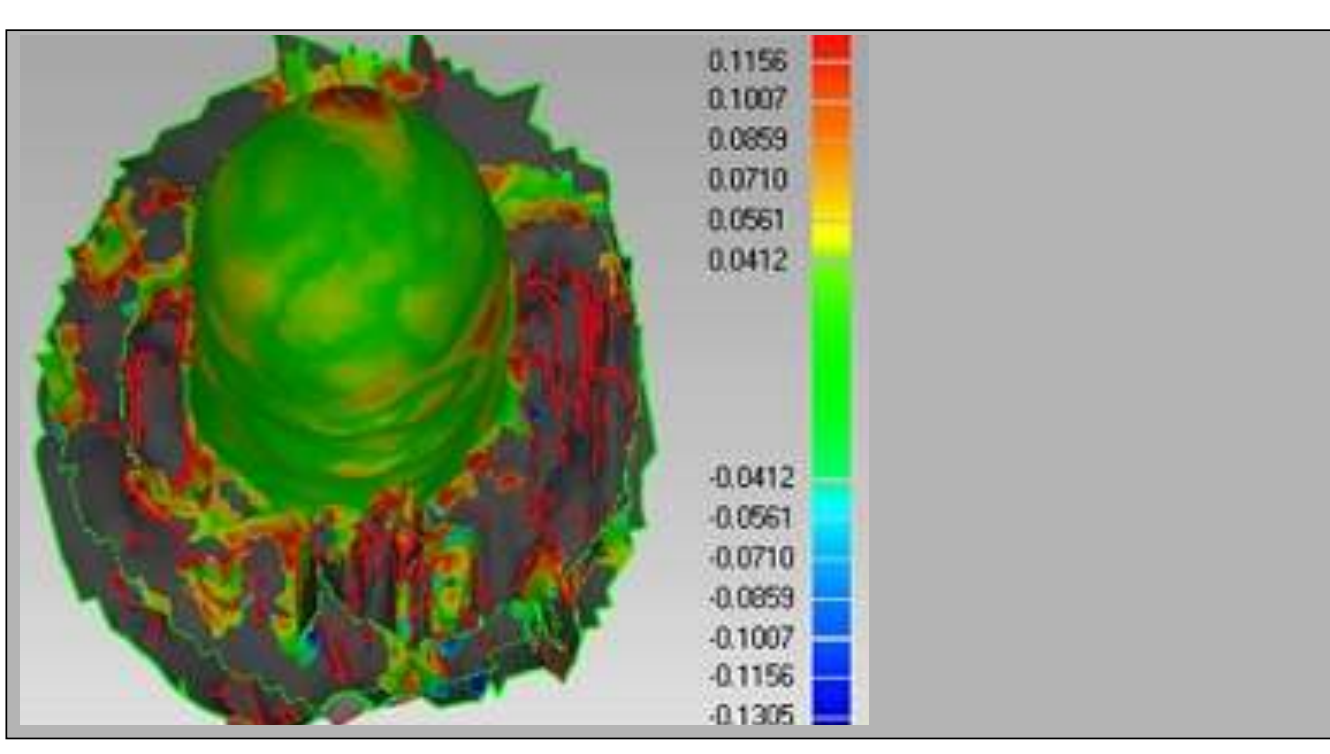

Figure 5: Correct image alignment. Color map: green mages for the bone perimeter closest to the implant are than 0.041 ned) (distance lower

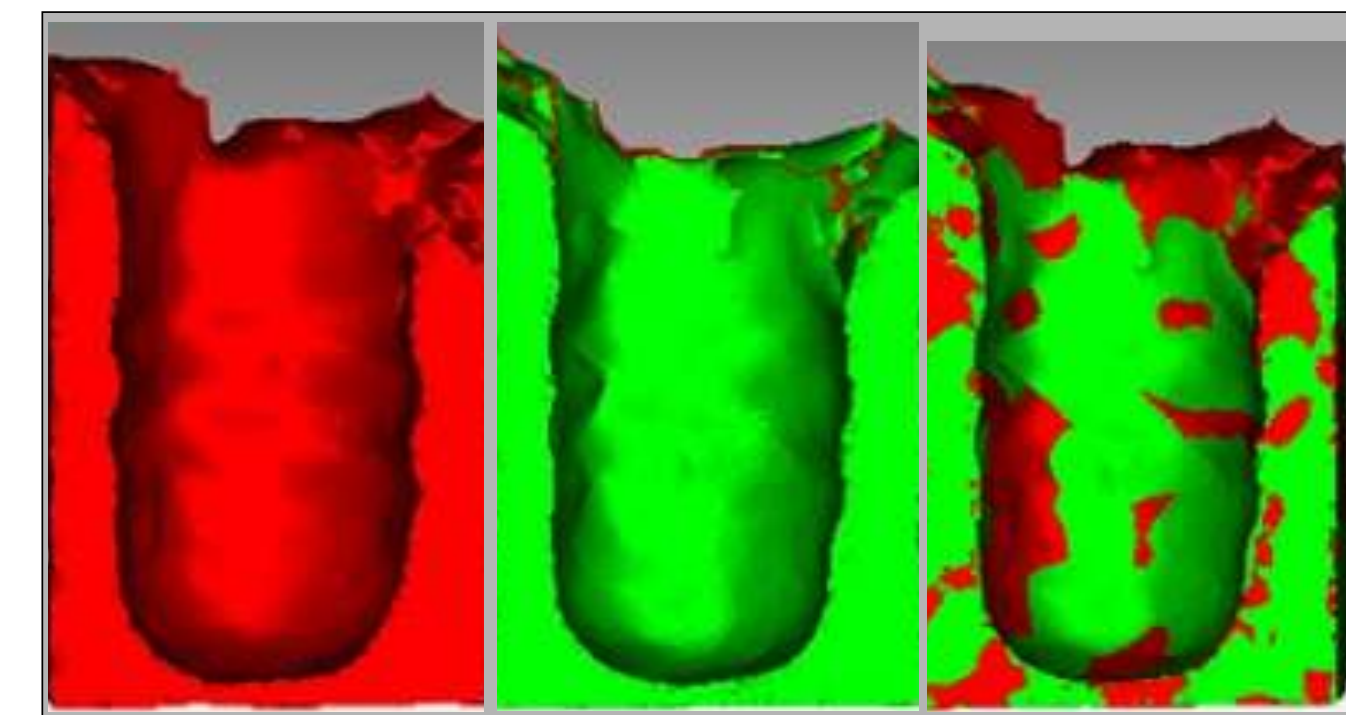

Figure 6: Images with cube wals al Indy projected, whe same for all times, and illustrating a possible cube section.

\section{Results}

- Deviations in methodology $\rightarrow$ some patients dismissed / excluded.

- Volumetric changes: $1^{\text {st }} \mathrm{yr} \rightarrow 30$ implants ; $2^{\text {nd }} \mathrm{yr} \rightarrow 34$ implants accumulated changes $1^{\text {st }} \& 2^{\text {nd }}$ yrs: 27 implants

- Mean peri-implant linear BL:

$$
1^{\text {st }} \mathrm{yr} \rightarrow 0.2 \pm 0.4 \mathrm{~mm} ; 2^{\text {nd }} \mathrm{yr} \rightarrow 0.1 \pm 0.2 \mathrm{~mm} \text {. }
$$

- Mean volumetric BL:

$$
1^{\text {st }} \mathrm{yr} \rightarrow 7.2 \pm 6,1 \mathrm{~mm}^{3} ; 2^{\text {nd }} \mathrm{yr} \rightarrow 6.4 \pm 7.8 \mathrm{~mm}^{3} \text {. }
$$

- 2D / 3D analysis estimated that an increase of $1 \mathrm{~mm}$ of linear BL was associated with a mean volumetric $B L$ of $\approx 14 \mathrm{~mm}^{3}(p<0.001)$.

\begin{tabular}{|c|c|c|c|}
\hline & 0-1 YEAR & 1-2 YEARS & 0-2 YEAR \\
\hline $\begin{array}{l}\text { LINEAR BONE LOSS } \\
(\mathrm{mm})\end{array}$ & $\begin{array}{c}0.2+-0.4(-0.4 ; 1.6) \\
n=45\end{array}$ & $\begin{array}{c}0.1+-0.2(-0.7 ; 0.4) \\
n=38\end{array}$ & $\begin{array}{c}0.2+-0.3(-0.6 ; 0.8) \\
n=38\end{array}$ \\
\hline $\begin{array}{l}\text { VOLUMETRIC } \\
\text { BONE LOSS }\left(\mathrm{mm}^{3}\right)\end{array}$ & $\begin{array}{c}7.2+-6.1(-0.5 ; 23.8) \\
n=30\end{array}$ & $\begin{array}{c}6.4+-7.8(-7.3 ; 29.0) \\
n=34\end{array}$ & $\begin{array}{c}12.6+-8.0(-1.7 ; 20.8) \\
n=27\end{array}$ \\
\hline
\end{tabular}

Table 1: Descriptive results of volumetric and linear bone loss

Table 2: Multilevel regression model to evaluate volumetric changes in bone level around dental implants in relation with linear measures

\begin{tabular}{|l|c|c|c|c|c|}
\hline PARAMETER & ESTIMATE & SE & \multicolumn{2}{|c|}{$95 \% \mathrm{CL}$} & $p$ \\
\hline INTERCEPT & 183.5758 & 8.7877 & 166.3523 & 200.7994 & $<.0001$ \\
\hline LINEAR BONE LOSS & 14.0200 & 3.2071 & 20.3059 & 7.7341 & $<.0001$ \\
\hline
\end{tabular}

\section{Conclusions}

- Measuring volumetric bone changes around implants is possible with СВCT. Proper contrast and sharpness on implant outline is mandatory.

- Improvements in image quality for bone tissue detection may allow a faster analysis to be easily applied in the dental office.

- Linear and volumetric bone loss measures are related. 